\title{
Bulk and surface localized modes on a magnetic nonlinear impurity
}

\author{
Mario I. Molina \\ Departamento de Física, Facultad de Ciencias, \\ Universidad de Chile, casilla 653, Santiago, Chile
}

\begin{abstract}
We study localized modes on a single magnetic impurity positioned in the bulk or at the surface of a one-dimensional chain, in the presence of a magnetic field $B$ acting at the impurity site. The strong on-site nonlinear interaction $U$ between two electrons of opposite spin at the impurity site, modelled here as a nonlinear local term, and the presence of the external field induce a strong correlation between parallel and anti-parallel spin bound states. We find that, for an impurity in the bulk, a localized vector mode (with up and down spin components) is always possible for any given value of $U$ and $B$, while for a surface impurity, a minimum value of both, $U$ and $B$ is needed to create a vector mode. In this case, up to two localized modes are possible, but only one of them is stable. The presence of the surface seems to destabilize the bulk mode in the parameter region $U \sim B$, creating a "forbidden strip" region in parameter space, bounded by $U=B+V$ and $U=B-V$, approximately.
\end{abstract}

PACS numbers: 71.55.-i, 72.10.Fk, 73.40.Gk 


\section{INTRODUCTION}

Nonlinear effects are the subject of intensive, ongoing research in several fields that include molecular crystals [1, 2], Josephson-junction arrays [3], Ferromagnetic materials [4], photonic crystals[5], photonic lattices[6], Bose-Einstein condensates in magneto-optical traps [7] and nonlinear metamaterials [8], to name a few. This interest is due in no small part to the wide range of potential applications to the design and operation of optoelectronic devices.

In condensed matter, nonlinear effects arise from at least two different sources. One of them is a possible strong coupling between an excitation and local vibrational modes. In the approximation where one assumes a rapid readjustments of the local vibrations to the presence of the excitation, one quickly arrives at some version of the discrete nonlinear Schrödinger (DNLS) equation, whose exact form depends on the type of anharmonicity (or lack of it) of the underlying oscillators[9]. When the strong interaction tales place only at some few sites, or at a single impurity site, it is possible to compute the formation of bound states in closed form in one-dimensional[10], two-dimensional[11] and threedimensional lattices [12], by using a direct extension of the lattice Green function formalism.

The other source for nonlinearity comes from electron-electron interactions in nanoscale devices, such as quantum dots and few impurity models [13]. Roughly speaking, the Coulomb interaction gives rise to a nonlinear term in the Schrödinger equation, that is usually modelled as a cubic, nonlocal term in the fermionic field operators. This is usually followed by the Hartree-Fock approximation for the nonlinear term[14], or the use of a perturbative approach in the Coulomb interaction[15]. Another recent approach calls for modelling

the electron-electron interaction by a nonlinear local term in the Schrödinger equation[16]. While being a sort of oversimplification of the many-body problem, it has the advantage of retaining the main features of Coulomb interaction-induced nonlinearities, while allowing for speedy computation of quantities of interest for electronic transport. This approach has been recently used for a simple computation of the zero-voltage conductance across a magnetic impurity[16].

In this Letter, we focus on the possible localized impurity modes that can reside on top of a nonlinear magnetic impurity, where the source of the nonlinearity is due to strong electronelectron interaction effects at the impurity site. It is important to ascertain the conditions under which such bound states exist, since they could, for instance, scatter other extended 
excitations in the system. The precise control of this scattering could be of importance in the control of the transport properties across nanoscale impurity regions.

\section{MODEL}

Let us start by considering the problem of a one-dimensional discrete system consisting of two linear chains (leads) joined by a strongly nonlinear magnetic region, where electronelectron effects are important. In the interacting region, the electron-electron repulsion is modelled by $U \rho_{0 \sigma} \rho_{0-\sigma}$, where $\rho_{0 \sigma}=\left|\psi_{0, \sigma}\right|^{2}$, where $\psi_{0, \sigma}$ is the probability amplitude of finding an electron of spin $\sigma=\uparrow, \downarrow$ on site $n=0$.

The coupled evolution equations for the probability amplitudes are

$$
\begin{aligned}
& i \frac{d \psi_{n, \uparrow}}{d t}+V\left(\psi_{n+1, \uparrow}+\psi_{n-1, \uparrow}\right)+\delta_{n, 0}\left(\epsilon_{0, \uparrow}+U\left|\psi_{n, \downarrow}\right|^{2}\right) \psi_{n, \uparrow}=0 \\
& i \frac{d \psi_{n, \downarrow}}{d t}+V\left(\psi_{n+1, \downarrow}+\psi_{n-1, \downarrow}\right)+\delta_{n, 0}\left(\epsilon_{0, \downarrow}+U\left|\psi_{n, \uparrow}\right|^{2}\right) \psi_{n, \downarrow}=0
\end{aligned}
$$

where $V$ is the nearest-neighbor coupling parameter, $U$ is the Coulomb repulsion energy and $\epsilon_{0, \uparrow}=-B, \epsilon_{0, \downarrow}=B$ is the Zeeman energy shift due to the magnetic field $B$, which is assumed to be appreciable only in the immediate vicinity of the impurity site.

We are interested in stationary solutions of the type $\psi_{n, \sigma}(t)=\exp (i \beta t) \psi_{n, \sigma}$. This leads to the system of nonlinear equations

$$
-\beta \psi_{n, \sigma}+V\left(\psi_{n+1, \sigma}+\psi_{n-1, \sigma}\right)+\delta_{n, 0}\left(\epsilon_{0, \sigma}+U\left|\psi_{n,-\sigma}\right|^{2}\right) \psi_{n, \sigma}=0
$$

At this point an general observation is in order. If we were to consider the impurity site also coupled to a local, fast elastic vibrational degree of freedom, then in the limit when the local vibration is completely enslaved to the electronic motion, there would be an additional term of the form $\gamma\left|\psi_{n, \sigma}\right|^{2} \psi_{n, \sigma}$ in Eqs.(1), (2). In that case, the system would be formally equivalent to a birefringent, nonlinear optical Kerr impurity embedded in a weakly-coupled, linear waveguide array, in the absence of four-wave effects, and $\psi_{n, \uparrow}$ and $\psi_{n, \downarrow}$ would represent the electric field amplitudes for the TM and TE mode, respectively[17].

In order to keep the magnetic and electronic repulsion effects well separated from possible polaronic effects, in this work we restrict ourselves to Eqs. (1), (2). The interplay of external magnetic field, electron-electron repulsion and electron-phonon interaction effects on a single impurity, will be described elsewhere. 

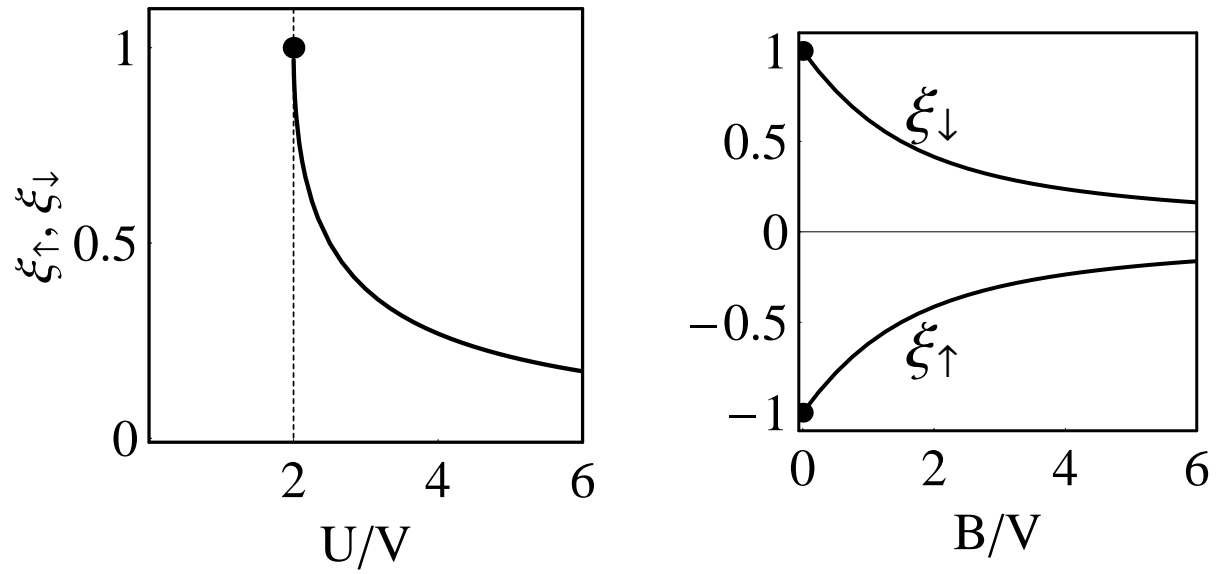

FIG. 1: (Color online). Left: $B=0$ case. Amplitude of localized state for both spins as a function of nonlinearity. Right: $U=0$ case. Amplitude of localized state for both spins, as a function of magnetic field.

\section{IMPURITY IN BULK}

Here the chain occupies the interval $-\infty<n<\infty$, with the impurity site at $n=0$. We look for a localized mode centered on the impurity site, $\psi_{n, \sigma}=A_{\sigma} \xi_{\sigma}^{|n|}$, with $0<|\xi|<1$. This ansatz leads to the equations:

$$
-\beta+2 V \xi_{\sigma}+\epsilon_{\sigma}+U A_{-\sigma}^{2}=0
$$

and

$$
\beta=V\left(\xi_{\sigma}+\frac{1}{\xi_{\sigma}}\right)
$$

On the other hand, from probability conservation, we have $1=\sum_{n}\left|\psi_{n, \uparrow}\right|^{2}=\sum_{n}\left|\psi_{n, \downarrow}\right|^{2}$. This implies the additional equations

$$
1=A_{\uparrow}^{2}\left(\frac{1+\xi_{\uparrow}^{2}}{1-\xi_{\uparrow}^{2}}\right)
$$

and

$$
1=A_{\downarrow}^{2}\left(\frac{1+\xi_{\downarrow}^{2}}{1-\xi_{\downarrow}^{2}}\right) .
$$

From Eqs.(3) $,(44),([5)$ and ([6)

$$
\xi_{\uparrow}^{2}=1-\xi_{\uparrow}\left[-\frac{B}{V}+\frac{U}{V}\left(\frac{1-\xi_{\downarrow}^{2}}{1+\xi_{\downarrow}^{2}}\right)\right]
$$



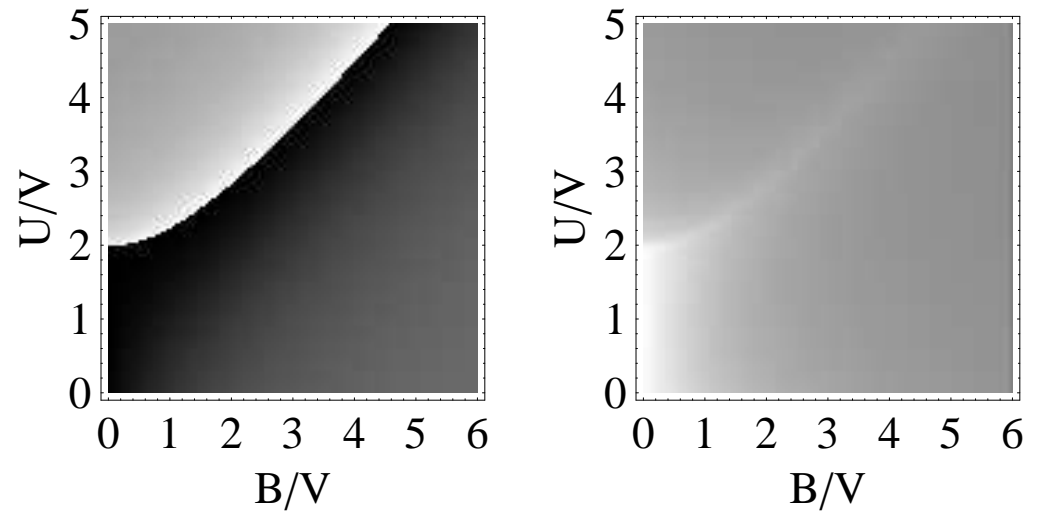

FIG. 2: Amplitude of localized mode as a function of nonlinearity and external magnetic field, for spin up (left) and spin down (right). Darkest (whitest) shade corresponds to $\xi=-1(1)$.

and

$$
\xi_{\downarrow}^{2}=1-\xi_{\downarrow}\left[+\frac{B}{V}+\frac{U}{V}\left(\frac{1-\xi_{\uparrow}^{2}}{1+\xi_{\uparrow}^{2}}\right)\right] .
$$

It is clear from these equations that, the transformation $\xi_{\uparrow} \rightarrow \xi_{\downarrow}, B \rightarrow-B$ leave the equations invariant. Since $U>0$ due to the repulsive nature of the Coulomb interaction, we only need to deal with, say, $B>0$. The behavior for the opposite $\operatorname{sign}$ of $B$ is obtained by simply exchanging $\xi_{\uparrow}$ and $\xi_{\downarrow}$.

Before analyzing the general case, let us discuss briefly a couple of important special cases.

(a) $\mathbf{B}=\mathbf{0}$. In this case, there is no physical distinction between $\xi_{\uparrow}$ and $\xi_{\downarrow}$, and Eqs.(7) and (8) collapse into a single one: $\xi^{2}=1+(U / V) \xi\left(\left(1-\xi^{2}\right) /\left(1+\xi^{2}\right)\right)$, with solution

$$
\xi=\left(\frac{U}{2 V}\right)-\sqrt{\left(\frac{U}{2 V}\right)^{2}-1}
$$

provided $U / V>2$. Since $\xi<0$, the spatial mode profile is staggered. Figure 1 shows $\xi$ in terms of $U / V$. As expected, an increase of nonlinearity reduces the width of the localized mode.

(b) $\mathbf{U}=\mathbf{0}$. In the absence of Coulomb repulsion, the only source for localization is given by the presence of the external field $B$. The equations read now $\xi_{\uparrow}^{2}=1-(B / V) \xi_{\uparrow}, \xi_{\downarrow}^{2}=$ $1+(B / V) \xi_{\downarrow}$, with solutions

$$
\xi_{\uparrow}=\left(\frac{B}{2 V}\right)-\sqrt{\left(\frac{B}{2 V}\right)^{2}+1}
$$



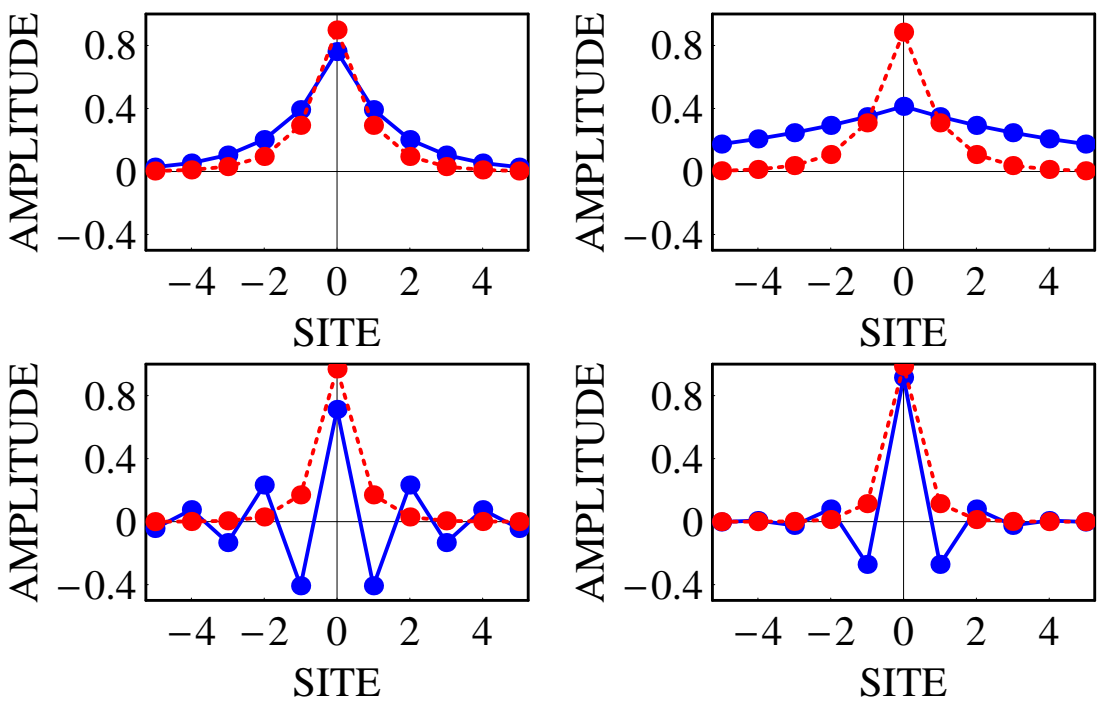

FIG. 3: (Color online). Spatial mode profiles for $U / V=3$ and varying values of $B / V$. Top left: $B / V=1$. Top right: $B / V=2$. Bottom left: $B / V=4$. Bottom right: $B / V=6$. Solid (dashed) curves denote the spin up (down) mode.

and

$$
\xi_{\downarrow}=-\left(\frac{B}{2 V}\right)+\sqrt{\left(\frac{B}{2 V}\right)^{2}+1} .
$$

Figure 1 shows the allowed $\xi_{\uparrow}, \xi_{\downarrow}$ as a function of magnetic field. In this case, no minimum field strength is needed to create a localized mode, but the mode profile corresponding to a spin parallel (antiparallel) to the external field is staggered (unstaggered).

For the general case, we solve Eqs.(7) and (8) numerically, selecting the real roots that lie in the interval $(-1,1)$. Results are shown on Fig.2 in the form of a nonlinearity-magnetic field phase space diagram, showing the amplitude of $\xi_{\uparrow}, \xi_{\downarrow}$, for a given value of $U / V$ and $B / V$. For spin up, the amplitude is always negative and increasing with increasing $B$, provided $U / V<2$. When $U / V>2$, there is a sharp boundary in $U-B$ space separating positive amplitude values, in the "low" $B / V$ region, from negative amplitude values, in the "high" $B / V$ value. Upon crossing the boundary, in the direction of increasing $B$, the sign of the amplitude jumps discontinuously from +1 to -1 . The shape of this critical boundary can be computed exactly from Eqs.(7) and (8): We set $\xi_{\uparrow}^{2}=1$ in Eq.(8), and solve for $\xi_{\downarrow}=-(B / 2 V)+\sqrt{(B / 2 V)^{2}+1}$. Next, we insert this into Eq.(7), and solve for $U$ in terms 
of $B$, obtaining:

$$
\left(\frac{B}{V}\right)_{c}=\frac{4+(B / V)^{2}-(B / V) \sqrt{(B / V)^{2}+4}}{-(B / V)+\sqrt{(B / V)^{2}+4}} .
$$

For spin down, the amplitude of the localized modes is always positive. For $U / V<2$, the amplitude decreases monotonically towards zero with an increase in $B$. For $U / V>2$ however, the amplitude starts at some positive value at $B=0$, then increases further with an increase in $\mathrm{B}$, until $B / V$ reaches the critical boundary computed above. After that, an increase in $B$ leads to an decrement of the amplitude towards zero.

In Fig.3 we show some spatial profiles $\psi_{n, \sigma}$ for a fixed value of nonlinearity, and increasing values of magnetic field. Note that for $B>0$, the localized mode for spin down is always unstaggered, while for spin up, the mode is unstaggered at first, and becomes staggered after certain value of magnetic field, given by Eq.(12).

\section{SURFACE IMPURITY}

We now consider the case when the magnetic impurity is at the very beginning of a semiinfinite lattice. We relabel the previous chain, so that the first site is now at $n_{0}=0$. The stationary-state equations read now

$$
\begin{gathered}
-\beta \psi_{0, \sigma}+V \psi_{1, \sigma}+\left(\epsilon_{0, \sigma}+U\left|\psi_{0, \sigma}\right|^{2}\right) \psi_{0, \sigma}=0, \\
-\beta \psi_{n, \sigma}+V\left(\psi_{n+1, \sigma}+\psi_{n-1, \sigma}\right)=0, \quad n=0,1,2, \ldots
\end{gathered}
$$

We proceed as before and pose a solution of the form $\phi_{n, \sigma}=A_{\sigma} \xi_{\sigma}^{n}$, where $0<|\xi|<1$ and $n=0,1,2, \ldots$ After replacing this ansatz into Eq.(13) and (14), one obtains $\beta=$ $V \xi_{\sigma}+\epsilon_{0, \sigma}+U A_{-\sigma}^{2}$ and $\beta=\xi_{\sigma}+\left(1 / \xi_{\sigma}\right)$, which implies

$$
\xi_{\sigma}=\frac{1}{\left(\epsilon_{\sigma} / V\right)+(U / V) A_{-\sigma}^{2}} .
$$

On the other hand, from the normalization condition, $1=\sum_{n}\left|\psi_{n, \uparrow}\right|^{2}=\sum_{n}\left|\psi_{n, \downarrow}\right|^{2}$, we obtain $A_{\uparrow}^{2}=1-\xi_{\uparrow}^{2}$ and $A_{\downarrow}^{2}=1-\xi_{\downarrow}^{2}$. After inserting this into Eq.(15) and after using $\epsilon_{0, \sigma}=-\sigma B$, one arrives at two coupled transcendental equations for $\xi_{\uparrow}, \xi_{\downarrow}$ :

$$
\begin{aligned}
\xi_{\uparrow} & =\frac{1}{-(B / V)+(U / V)\left(1-\xi_{\downarrow}^{2}\right)}, \\
\xi_{\downarrow} & =\frac{1}{(B / V)+(U / V)\left(1-\xi_{\uparrow}^{2}\right)} .
\end{aligned}
$$



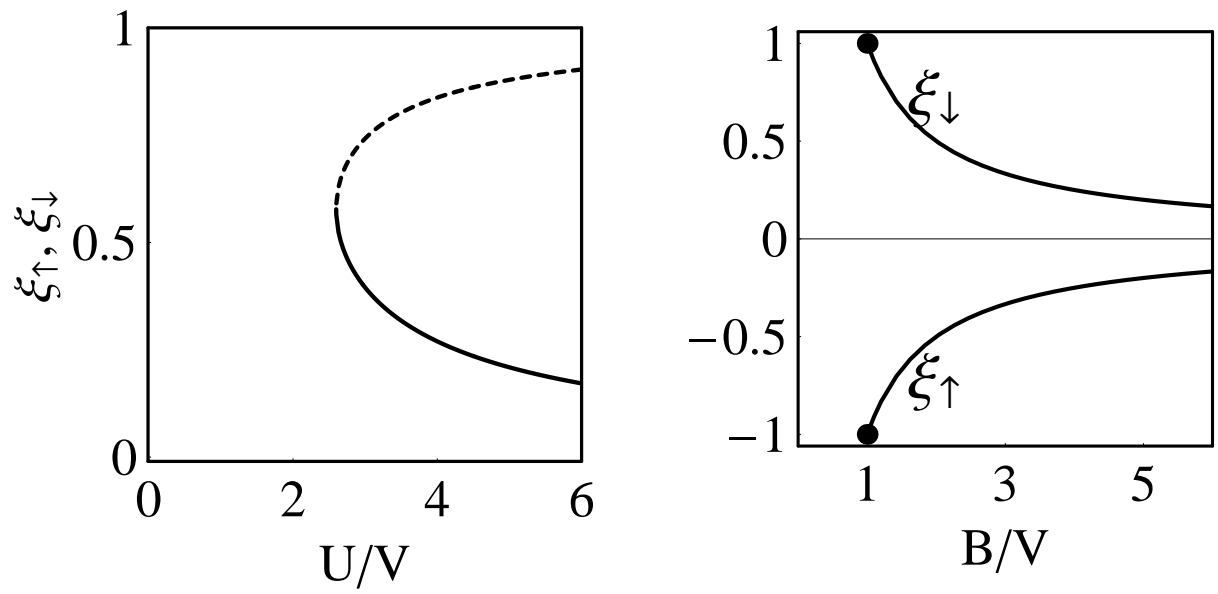

FIG. 4: Left: $B=0$ case. Amplitude of localized state for both spins as a function of nonlinearity. The solid (dashed) curve denotes the stable (unstable) mode. Right: $U=0$ case. Amplitude of localized state for both spins, as a function of magnetic field.

As was done for the impurity in the bulk, let us consider first two special cases.

(a) $\mathbf{B}=\mathbf{0}$. In this case, $\xi_{\uparrow}=\xi_{\downarrow}=\xi$, where $\xi$ satisfies $\xi\left(1-\xi^{2}\right)=(U / V)^{-1}$. This implies that a critical value $(U / V)_{c}=(3 / 2) \sqrt{3} \sim 2.6$ exists, such that for $(U / V)<(U / V)_{c}$, no localized state exists. For $(U / V)=(U / V)_{c}$, there is exactly one localized state, and $(U / V)>(U / V)_{c}$, two localized states are possible; for one of them, $\xi$ increases towards unity as nonlinearity is increased (unstable mode), while the other decreases $\xi$ as nonlinearity is increased (stable mode). Since $U>0$, this implies that $\xi$ is also positive, and thus, the localized mode is unstaggered. Figure 4 shows $\xi$ in terms of $U / V$.

(b) $\mathbf{U}=\mathbf{0}$. In the absence of Coulomb repulsion, we immediately obtain $\xi_{\uparrow}=-1 /(B / V)$ and $\xi_{\downarrow}=1 /(B / V)$, where $|B / V|>1$ to ensure $\left|\xi_{\uparrow, \downarrow}\right|<1$. Figure 4 shows the allowed $\xi_{\uparrow}, \xi_{\downarrow}$ as a function of magnetic field. The mode profile corresponding to a spin parallel (antiparallel) to the external field is staggered (unstaggered).

Although Figs. 1 and 4 look qualitatively similar, we note some interesting differences: For the surface impurity, and in the absence of magnetic field, the amount of nonlinearity needed to effect a localized state is higher that in the bulk impurity case. Also, in the linear case $(U=0)$, one needs higher values of magnetic field to create a surface localized mode. The surface, or boundary of the system is acting in a repulsive manner.

For the general case, there are two important regimes, $B / V \leq 1$ and $B / V>1$.

$B / V \leq 1$ : In this case, there is a critical nonlinearity value $(U / V)_{c}$ such that, for $0<$ 
$(U / V)<(U / V)_{c}$, no localized state exists, while for $(U / V)>(U / V)_{c}$, there are two localized states. One of them (stable mode) becomes narrower with increasing nonlinearity; the other (unstable mode) becomes wider.

$B / V>1$ : In this case, we have three critical values for $(U / V)$, that create four regions: (i) $0<(U / V)<(U / V)_{c 1}$ : This is the region of "small" nonlinearity, where magnetic field dominates and there is always a localized mode, with $\xi_{\uparrow}<0, \xi_{\downarrow}>0$. As $(U / V) \rightarrow(U / V)_{c 1}$, $\xi_{\uparrow} \rightarrow-1$.

(ii) $(U / V)_{c 1}<(U / V)<(U / V)_{c 2}$ : No localized mode exists.

(iii) $(U / V)_{c 2}<(U / V)<(U / V)_{c 3}$ : Two localized modes are possible here. One of them (stable) becomes narrower with increasing $U / V$, while the other (unstable) becomes wider with increasing $U / V$.

(iv) $(U / V)>(U / V)_{c 3}$ : The previous unstable state disappears, and only stable mode remains. As $(U / V) \rightarrow \infty$, both $\xi_{\uparrow}$ and $\xi_{\downarrow}$ approach zero.

Figure 5 shows all these regions in the form of a nonlinearity-magnetic field phase space diagram. The number inside each region denotes the number of localized surface modes (each one with a spin up and spin down component). We note, in particular, the existence of a region, roughly delimited by the straight lines $(U / V)=(B / V)+1$ and $(U / V)=(B / V)-1$, where no localized states exists. These are obtained as limiting cases of the curves that separate regions "1" (top) and "0", and regions "0" and "1" (bottom). For the first case the curve is $U / V=[(B / V)+1] /\left[1-(V / B)^{2}\right]$, while for the second case, the curve is given by $U / V=[(B / V)-1] /\left[1-(V / B)^{2}\right]$. In Fig. 6 we show examples of spatial profiles $\psi_{n, \sigma}$. We nota that their staggered/unstaggered character is rather similar to the "bulk" case (Fig.3).

\section{SELFTRAPPING DYNAMICS}

We consider now the dynamical excitation of a localized mode on top of the magnetic impurity, comparing the bulk and surface cases. We place initially both electrons (with opposite spins) on the impurity site and follow the evolution of their probability densities, according to Eq.(1). In particular, we focus on the time-averaged selftrapped fraction remaining the initial site, defined by

$$
\left\langle P_{\sigma}\right\rangle=\frac{1}{T} \int_{0}^{T}\left|\xi_{\sigma}(t)\right|^{2} d t,
$$




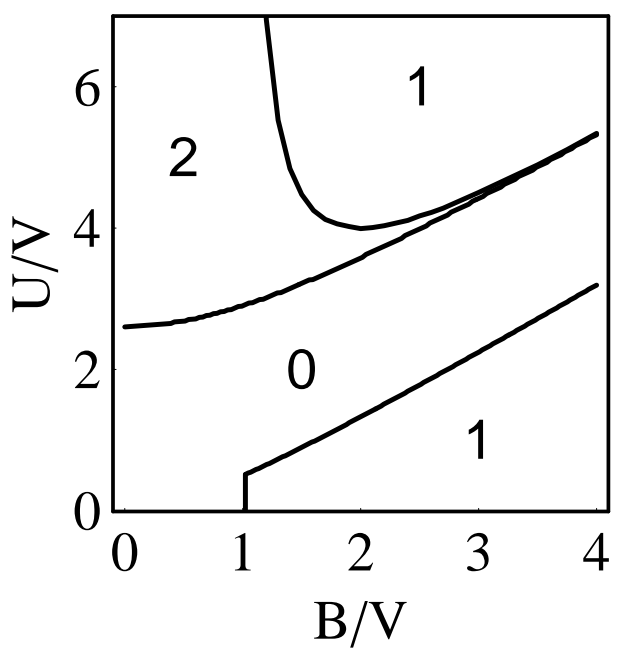

FIG. 5: Number of localized surface modes, as a function of nonlinearity and magnetic field.
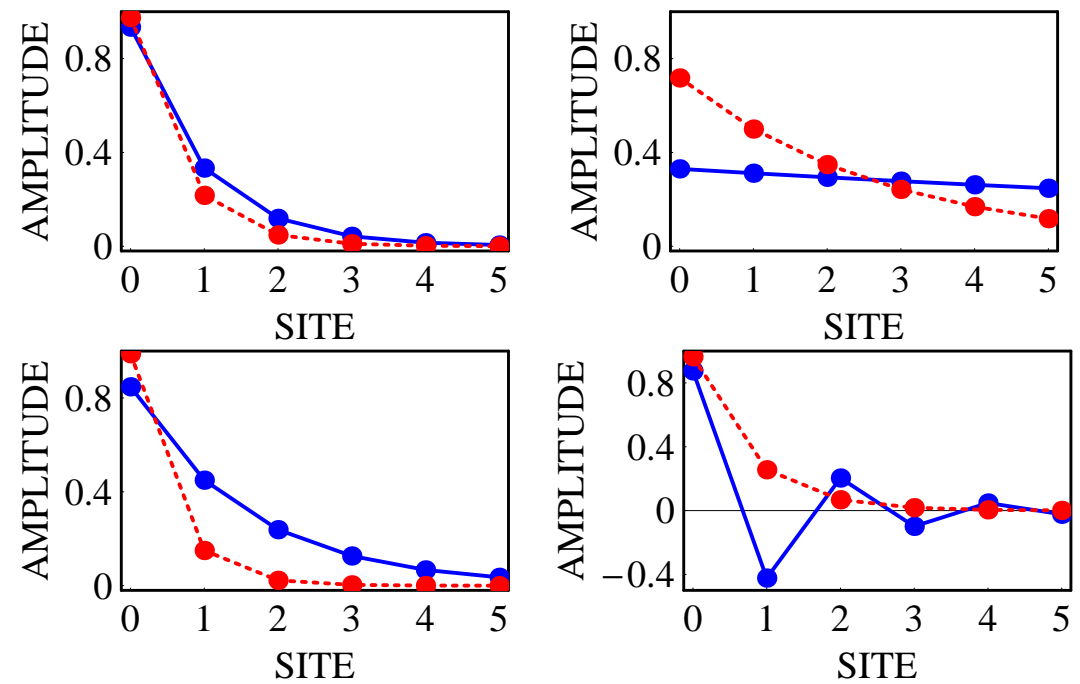

FIG. 6: (Color online). Examples of surface localized modes. Solid (dashed) lines refer to spin up (down). Top left: $B / V=1, U / V=4$ (stable mode). Top right: $B / V=1, U / V=4$ (unstable mode). Bottom left: $B / V=3, U / V=5$. Bottom right: $B / V=3, U / V=1$.

and examine how $\left\langle P_{\sigma}\right\rangle$ depends on electron interaction $U$ and magnetic field $B$.

Figures 7 and 8 show the selftrapping results for the "bulk" and surface cases. While the behavior in both cases is qualitatively similar, we note that in general, it is a bit easier to selftrap in the bulk case than in the surface case. On the other hand, for the surface case, there are sharper boundaries separating the untrapped from the selftrapped regime. Also, in both cases and for positive $B$, selftrapping for the spin up component, is largely inhibited 
in a wide strip around the region $U \sim B$, while the spin down component is dominated by magnetic field effects. Of course, for $U<<B$, nonlinearity effects are not important and selftrapping increases gradually with magnetic field, as one expects for a linear site impurity. On the contrary, for $U>>B$, selftrapping is abrupt and its threshold is higher for the surface case than for the bulk case. Again, this is a manifestation of the repulsive nature of the system surface in one-dimension.
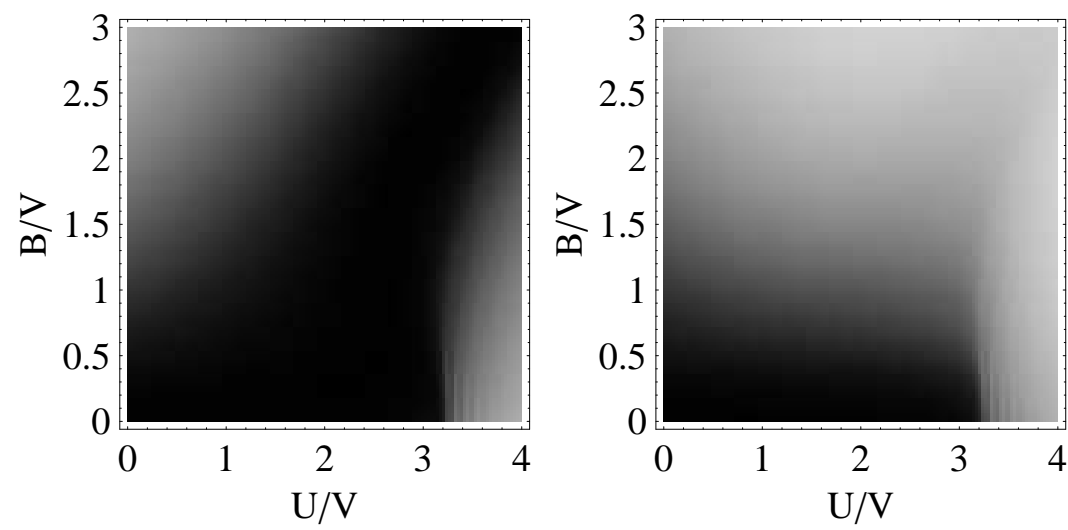

FIG. 7: Magnetic impurity in "bulk": Left (right): Time-averaged selftrapped fraction of spin up (down) remaining on the initial site, as a function of $U$ and $B$. Dark (white) shade denote selftrapped fraction close to zero (one).
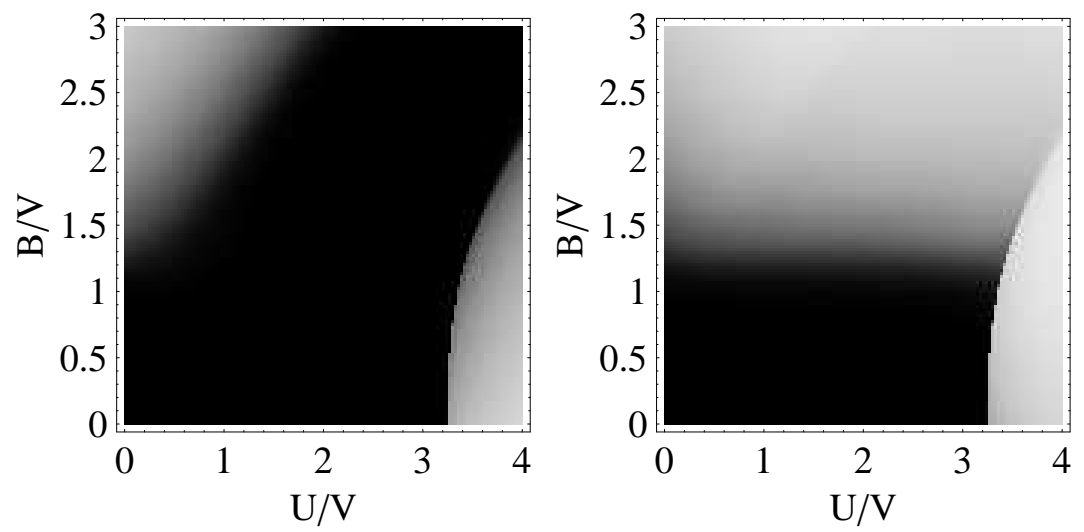

FIG. 8: Magnetic impurity on surface: Left (right): Time-averaged selftrapped fraction of spin up (down) remaining on the initial site, as a function of $U$ and $B$. Dark (white) shade denote selftrapped fraction close to zero (one). 


\section{CONCLUSIONS}

We have examined theoretically the formation of bound spin states on top of a narrow magnetic impurity region in the presence of an external magnetic field, in the simplified framework of modelling the electronic repulsion by means of a local nonlinear term. The ensuing set of coupled DNSL equations obtained for the vector impurity, is solved numerically for arbitrary values of electronic repulsion and magnetic field strength. We focused on the cases of a bulk and a surface impurity. In general, we found that it is easier to create a bound state in the bulk case than in the surface case. In the latter, the bound state diagram showing the number of bound states in terms of nonlinearity and magnetic field parameters, show a rather complex structure, that includes a "forbidden region" delimited approximately by $U=B+V$ and $U=B-V$. In this regard, the presence of a surface inhibits completely the creation of a vector localized mode, in the region $U \sim B$. In addition, there is a parameter region where two bound states exist, although only one of them is (linearly) stable. Dynamical results for selftrapping show that, in general, it is easier to selftrap on a bulk impurity than on a surface one, although the selftrapping is sharper for the latter. This repulsive character of the surface was observed earlier for the one-dimensional nonlinear chain [18], and seems to be generic to one-dimensional discrete systems.

\section{ACKNOWLEDGEMENTS}

This work has been supported by Fondecyt Grant 1080374. 
[1] A. J. Sievers and S. Takeno, Phys. Rev. Lett. 61, 970 (1988).

[2] See, for instance, D. K. Campbell, S. Flach and Y. S. Kivshar, Phys. Today 57, 43 (2004).

[3] P. Binder, D. Abraimov, A. V. Ustinov, S. Flach and Y. Zolotaryuk, Phys. Rev. Lett. 84, 745 (2000);

E. Trias, J. J. Mazo and T. P. Orlando, Phys. Rev. Lett. 84, 741 (2000);

J. J. Mazo, Phys. Rev. Lett. 89,234101 (2002).

[4] U. T. Schwarz, L. Q. English, and A. J. Sievers, Phys. Rev. Lett.89, 223 (1999).

[5] M. Yanik, S. Fan, M. Soljacic and J. D, Joannopoulos, Opt. Lett. 28, 2506 (2003);

D. Christodoulides and N. K. Efremidis, Opt. Lett. 27, 568 (2002);

S. F. Mingaleev and Y. S. Kivshar, Phys. Rev. Lett. 86, 5474 (2001);

S. F. Mingaleev and Y. S. Kivshar, Opt. Lett. 27, 231 (2202).

[6] J. W. Fleischer, M. segev, N. K. Efremidis and D. Christodoulides, Nature 422, 147 (2003);

J. W. Fleischer, T. Carmon, M. Segev, N. K. Efremidis and D. Christodoulides, Phys. rev. Lett.90, 023902 (2003);

N. K. Efremidis, J. Hudock, D. Christodoulides, J. Fleischer, O. Cohen and M. Segev, Phys. Rev. Lett. 91, 213906 (2003).

[7] L. Khaykovich, F, Schreck, G. Ferrari, T. Bourdel, J. Cubizolles, L.D. Carr, Y. Castin and C. Solomon, Science 296, 1290 (2002);

A. Trombettoni and A. Smerzi, Phys. Rev. Lett. 86, 2353 (2001);

[8] Nikos Lazarides, George P. Tsironis, and Yuri S. Kivshar, Phys. Rev. E 77, 065601 (2008);

A. Maluckov, Lj. Hadžievski, N. Lazarides, and G. P. Tsironis, Phys. Rev. E 77, 046607 (2008);

Yongmin Liu, Guy Bartal, Dentcho A. Genov, and Xiang Zhang, Phys. Rev. Lett. 99, 153901 (2007);

Natalia M. Litchinitser, Ildar R. Gabitov, and Andrei I. Maimistov, Phys. Rev. Lett. 99, 113902 (2007).

[9] D. Chen, M. I. Molina and G. P. Tsironis, J. Phys. Condens. Matter 5, 8689 (1993).

[10] M. I. Molina and G. P. Tsironis, Phys. Rev. B 47, 15330 (1993).

[11] M. I. Molina, Phys. Rev. B 60, 2276 (1999).

[12] C. A. Bustamante and M. I. Molina, Phys. rev. B 62, 15287 (2000). 
[13] N. F. Johnson, J. Phys. Condens. Matter 7, 965 (1995).

[14] S. M. Apenko, Ya. M. Blanter, Yu. E. Losovik, Phys. Lett. A 176, 137 (1993).

[15] A. Oguri, Phys. Rev. B 59, 12240 (1999).

[16] M. I. Molina and H. bahlouli, Phys. Lett. A 294, 87 (2002).

[17] See, for instance, J. Meier, J. Hudock, D. Christodoulides, G. Stegeman, Y. Silberberg, R. Morandotti and J. S. Aitchison, Phys. Rev. Lett. 91, 143907 (2003), and references therein.

[18] M. I. Molina, R. A. Vicencio and Y. S. Kivhar, Opt. Lett. 31, 1693 (2006). 


\section{List of Figure Captions}

Figure 1: Left: $B=0$ case. Amplitude of localized state for both spins as a function of nonlinearity. Right: $U=0$ case. Amplitude of localized state for both spins, as a function of magnetic field.

Figure 2: Amplitude of localized mode as a function of nonlinearity and external magnetic field, for spin up (left) and spin down (right). Darkest (whitest) shade corresponds to $\xi=-1(1)$.

Figure 3: (Color online). Spatial mode profiles for $U / V=3$ and varying values of $B / V$. Top left: $B / V=1$. Top right: $B / V=2$. Bottom left: $B / V=4$. Bottom right: $B / V=6$. Solid (dashed) curves denote the spin up (down) mode.

Figure 4: Left: $B=0$ case. Amplitude of localized state for both spins as a function of nonlinearity. Right: $U=0$ case. Amplitude of localized state for both spins, as a function of magnetic field.

Figure 5: Number of localized surface modes, as a function of nonlinearity and magnetic field.

Figure 6: (Color online). Examples of surface localized modes. Solid (dashed) lines refer to spin up (down). Top left: $B / V=1, U / V=4$ (stable mode). Top right: $B / V=1, U / V=4$ (unstable mode). Bottom left: $B / V=3, U / V=5$. Bottom right: $B / V=3, U / V=1$

Figure 7: (Color online). "Bulk" magnetic impurity: Time-averaged selftrapped fraction for both spins remaining on the initial site, as a function of $U$ and $B$.

Figure 8: (Color online). Surface magnetic impurity: Time-averaged selftrapped fraction for both spins remaining on the initial site, as a function of $U$ and $B$. 